\title{
A Risk-Neutral Consumer and His Coherent Decisions Under Uncertainty: A Methodological Analysis
}

\author{
Pierpaolo Angelini ${ }^{1}$ \\ ${ }^{1}$ Università La Sapienza di Roma, Dipartimento di Scienze Statistiche, Roma, Italia \\ Correspondence: Pierpaolo Angelini, Università La Sapienza di Roma, Dipartimento di Scienze Statistiche, \\ Roma, Italia. Tel: 39-064-9911. E-mail: pier.angelini@uniroma1.it
}

Received: January 26, 2020

Accepted: March 12, 2020

Online Published: March 30, 2020

doi:10.5539/ijef.v12n4p95

URL: https://doi.org/10.5539/ijef.v12n4p95

\begin{abstract}
If we study the expected utility function then we deal with a unified approach to an integrated formulation of decision theory in its two subjective components: utility and probability. We decompose the expected utility function inside of an $m$-dimensional linear space after decomposing a contingent consumption plan viewed as a univariate random quantity. We propose a condition of coherence compatible with all possible attitudes in the face of risk of a consumer. It is a geometric condition of coherence. In particular, we consider a risk-neutral consumer and his coherent decisions under uncertainty. The right closed structure in order to deal with utility and probability is a linear space in which we study coherent decisions under uncertainty having as their goal the maximization of the prevision of the utility associated with a contingent consumption bundle.
\end{abstract}

Keywords: collinearity, risk, expected utility function, random process, direct and orthogonal sum, contingent consumption bundle

\section{Introduction}

We study different and objectively possible states of nature, where each of them is expressed by a real number. We consider incompatible and exhaustive states of nature whose number is finite. One and only one of them will be true a posteriori. A state of nature is objectively possible for a given consumer with a given set of information at a given instant when he does not know whether it will definitively be true or false. We therefore say that an objectively possible state of nature is uncertain for this reason. A possible state of nature is nothing but a random event coinciding with a proposition. Any proposition is always expressed in an unambiguous way. When we speak about a random quantity we mean that it admits two or more than two objectively possible values. They are real numbers. When we speak about a univariate random quantity we mean that a real number always coincides with an objectively possible value of it. The set of all objectively possible alternatives of a univariate random quantity $X$ is denoted by

$$
\left\{x^{1}, x^{2}, \ldots, x^{\mathrm{m}}\right\}
$$

where we consider $x^{1}<x^{2}<\ldots<x^{m}$ without loss of generality.

A univariate random quantity is denoted by $X$. One and only one objectively possible value of $X$ will be true a posteriori. All other values will be false. It is possible to assign a probability to every $x_{i}, i=1, \ldots, m$, before knowing this thing. We obtain a distribution of probability in this way. A contingent consumption bundle is nothing but a univariate random quantity. It is denoted by

$$
\left[\left(x^{1}, P_{1}\right),\left(x^{2}, P_{2}\right), \ldots,\left(x^{m}, P_{m}\right)\right]
$$

in this way, where it turns out to be $p_{1}+p_{2}+\ldots+p_{m}=1$. Every single random case is generically denoted by $E_{i}$, $i=1, \ldots, m$. After decomposing $x^{l}, x^{2}, \ldots, x^{\mathrm{m}}$ inside of an m-dimensional linear space denoted by $E^{m}$ we observe that it is possible to assign a number to every element $x^{i}, i=1, \ldots, m$, of X. It is denoted by $u\left(x^{i}\right), i=1, \ldots, m$, where it turns out to be $u\left(x^{l}\right)<u\left(x^{2}\right)<\ldots<u\left(x^{m}\right)$ because one assumes that more is better when there is no satiation. All numbers expressed by $u\left(x^{i}\right), i=1, \ldots, m$, identify a utility function (Levy \& Sarnat, 1986). It is possible to associate a probability with every $u\left(x^{i}\right), i=1, \ldots, m$. We obtain a distribution of probability in this way. Every distribution of probability is always a coherent expression of the attitude of the consumer under consideration with respect to uncertainty (De Finetti, 1972). It does not depend on one or more than one parameter like a pre-established distribution (De Finetti, 1972). This means that one engages oneself in saying all 
what it is of interest about the specific case that is considered (Kreps, 1990). Thus, one does not prefer to race on ahead occupying oneself with not real problems characterized by infinite and repeatable cases. On the other hand, it is known that the nature of probability is unitary in all fields (Pfanzagl, 1967). Nevertheless, information used in order to make a coherent evaluation of probability related to a given set of states of nature can be different with respect to its external aspects. This implies that the criteria for the evaluation of probability are different (Taylor, 1974). However, they lead to an evaluation which is always subjective. This is because an equiprobable judgment is itself subjective. Such a judgment intrinsically characterizes symmetric probability. Concerning frequentist probability, it makes sense that each individual relates probability back to observed frequency only when he specifies the meaning and conditions of this thing. Symmetric probabilities as well as frequentist probabilities are only elements of judgment evaluated by each individual on the basis of his own judgment. Subjective probability results from this necessary judgment. In any case, it is not excluded that subjective probability coincides with symmetric probability. It is not even excluded that subjective probability coincides with frequentist probability. We do not refer to the axiomatic probability theory, so the field over which probability is defined is not a $\sigma$-algebra (Berti, Pratelli, \& Rigo, 2015).

\section{A Dichotomy between Possibility and Probability}

We consider those situations of uncertainty in which a given consumer can find himself because his information and knowledge are imperfect at a given instant (Feller, 1966). A given consumer is in a state of uncertainty at a given instant when he is in a state of ignorance (DeGroot, 1962). It is the result of the existence of imperfect information and knowledge at a given instant (Piccinato, 1986). Such an imperfection could be related to different facts or events. We deal with future facts within this context (Barlow, Claroti, \& Spizzichino, 1993). If the imperfect knowledge and the ignorance of a given consumer at a given instant determine his state of uncertainty then such a state represents the extent of his domain of the possible. It is objective because it is relative to his "state of information". Such a domain contains all possible states of nature for him at a given instant. We say that the states of nature are points belonging to an $m$-dimensional linear space equipped with a Euclidean metric. We denote it by $E^{m}$. Given an orthonormal basis of $E^{m}$ denoted by $\left\{e_{j}\right\}, j=1, \ldots, m$, we are able to consider $m$ oriented straight lines of $E^{m}$ which are measured in the same unit of length. They are pairwise orthogonal. The point where they meet is the origin of $E^{m}$. It is the zero vector of $E^{m}$. We say that each $x, i=$ $1, \ldots, m$, of $\left\{x, x, \ldots, x^{m}\right\}$ belongs to one of these $m$ oriented straight lines of $E$. We do not consider particular $m$-tuples of real numbers belonging to every straight line of $E^{m}$ but we consider only real numbers connected with each of them. This thing results from a particular geometric property that we will later use. Each straight line of $E^{m}$ represents the whole of the space of alternatives whose number is infinite. Each point on a straight line of $E^{m}$ corresponds to a single alternative and vice versa. Information and knowledge of a given consumer at a given instant permit him of not to excluding a real number only. It remains possible for him because it is not either true or false (Coletti, Petturiti, \& Vantaggi, 2015). It is denoted by $x^{i}, i=1, \ldots, m$. We have evidently a limitation of expectations in this way. We distinguish what it is determined from what it is not determined (De Finetti, 1982). We use geometry in order to make this thing (Ramsey, 1960). What it is not determined it is possible (Elton \& Gruber, 1995). What it is possible it is uncertain because it is not either true or false. It is an intermediate case with respect to what it is determined (Jeffreys,1961). It interests the notion of probability. Subjective probabilities are assigned to every $x^{i}, i=1, \ldots, m$, of $\left\{x^{1}, x^{2}, \ldots, x^{m}\right\}$ (Kyburg jr., \& Smokler, 1964). Also, we note that subjective utilities are assigned to every $x^{i}, i=1, \ldots, m$, of $\left\{x^{1}, x^{2}, \ldots, x^{m}\right\}$. What it is possible it interests the notion of utility as well.

\section{A Closed Structure Containing States of Nature as a Substitute for Another Closed Structure}

A state of nature is an event. An event is a proposition (Coletti \& Scozzafava, 2002). It is always a well-determined proposition in itself. A random event is always a single random case. It must always be observed by a given individual in order to establish whether it is true or false (Savage, 1954). A probability is then assigned to it by him before knowing this thing (Lindley, 1965). We say that a probability is assigned to it by him according to his degree of belief in the occurrence of it. It is possible to measure this degree of belief in a concrete or operational way. It is possible to observe which real or conceptual bets on a random event are judged to be fair by a given individual with a given set of information at a given instant. We note that those bets in which a given individual with a given set of information at a given instant accepts to be either bettor or bookmaker are judged to be fair by him (De Finetti, 1980). Probability is always defined into the domain of events (De Finetti, 1981). We note that if we consider a conditional bet based on a conditional event then the main points of what we have said do not change (Gilio \& Sanfilippo, 2014). We observe only formal variations with respect to a conditional bet based on a conditional event. These variations are conceptually unimportant 
(Coletti, Petturiti, \& Vantaggi, 2016). We are not evidently interested in using a metaphysical notion of probability of a random event within this context (Kendall \& Stuart, 1961). If we say that probability exists on its own because it does not depend on the mental or instinctive evaluations that a given individual makes of a given random event at a given instant with a given set of information then we use a metaphysical notion of probability. If we say that probability exists outside of him in the sense that it does not exist in his own judgment then we use a metaphysical notion of probability. If we say that probability must externally be represented like something acting behind the universe which can be observed according to its own axioms then we use a metaphysical notion of probability (Koopman, 1940). It follows that a random event does not belong to a larger closed structure containing different subsets of a given nonempty set (Good, 1962). A state of nature viewed as a possible proposition is conversely contained in another closed structure. Such an algebraic structure is not a $\sigma$-algebra where countable additivity unconditionally holds. It is a linear space over the field $R$ of real numbers where we consider finite additivity as a condition of coherence. We say that a state of nature has an intrinsic meaning because we do not choose a particular orthonormal basis of the linear space under consideration among all its possible orthonormal bases. We consider a linear space over $\mathrm{R}$ because a state of nature is a random entity that is expressed by numbers. It definitively admits only two values coinciding with two different numbers. If a state of nature is definitively true then its value is numerically expressed by 1 . If a state of nature is definitively false then its value is numerically expressed by 0 . We are therefore able to give to the states of nature an arithmetic interpretation in this way. Such an interpretation is a linear interpretation.

\section{Distributions of Probability Embedded in a Linear Space}

A contingent consumption plan is a list of $\mathrm{m}$ different real numbers that tells us how much a given consumer consumes of $X, x^{i}$, when $E_{i}$ occurs, where $E_{i}$ is the single state of nature associated with $x^{i}, i=1, \ldots, m$. It is geometrically identified with the components of an m-dimensional vector of an m-dimensional linear space denoted by $E^{m}$. Let $\left\{e_{j}\right\}$ be $, j=1, \ldots, m$, an orthonormal basis of $E^{m}$. If we use the Einstein summation convention then we write

$$
x=x^{i} e_{i}
$$

with $\mathrm{x} \in E^{m}$ (McCullagh, 1987). We observe that the possible values of $\mathrm{X}$ coincide with $x^{i}, i=1, \ldots, m$. They are different from one another because we deal with a partition of incompatible and exhaustive events (Mas-Colell, Whinston, \& Green, 1995). A contingent consumption plan is an element of a set of contingent consumption plans denoted by ${ }_{(1)} \mathrm{S}$. We note that it turns out to be

$$
{ }_{(1)} \mathrm{S} \subset E^{m}
$$

where ${ }_{(1)} \mathrm{S}$ is an m-dimensional linear space contained in $E^{m}$. This is because the sum of two vectors belonging to ${ }_{(1)} \mathrm{S}$ must be a vector whose components are all different. Thus, it belongs to ${ }_{(1)} \mathrm{S}$ in this way. We say that it belongs to ${ }_{(1)} \mathrm{S}$ if and only if its components are all different. The same thing goes when we consider the multiplication of a vector of ${ }_{(1)} \mathrm{S}$ by a real number that is different from zero. Hence, we say that ${ }_{(1)} \mathrm{S}$ is closed with respect to the sum of two vectors of it and the multiplication of a vector of it by a real number that is different from zero. We note that $E^{m}$ can also be viewed as an affine space over itself. Each element of $E^{m}$ is firstly an m-dimensional vector viewed as an ordered list of $\mathrm{m}$ real numbers. Nevertheless, each element of Em can also be viewed as a point of an affine space, where the zero vector of $E^{m}$ is the origin of it. Thus, the zero vector of $E^{m}$ characterizes an affine frame of $E^{m}$ when it is viewed as an affine space. An affine frame of $E^{m}$ viewed as an affine space consists of a point coinciding with the zero vector of $E^{m}$ and an orthonormal basis of $E^{m}$. We are able to consider a point of an affine space having $m$ coordinates or a vector of a linear space having $m$ components. The possible values of $X$ can indifferently be denoted by a covariant or contravariant notation after choosing an orthonormal basis of $E^{m}$. We should exactly speak about components of $x$ having upper orlower indices because we deal with an orthonormal basis of $E^{m}$. Indeed, to use the terms covariant and contravariant is geometrically meaningless because the covariant components of $x$ coincide with the contravariant ones. Nevertheless, it is appropriate to use this notation because a particular meaning connected with these components will be introduced. Thus, we choose a contravariant notation with respect to the components of $\mathrm{x}$. It is consequently possible to write

$$
\mathbf{x}=\left(\begin{array}{c}
x^{1} \\
x^{2} \\
\vdots \\
x^{m}
\end{array}\right) .
$$

We choose a covariant notation with respect to the components of $p \in E^{m}$. It is therefore possible to write 


$$
\mathbf{p}=\left(\begin{array}{c}
p_{1} \\
p_{2} \\
\vdots \\
p_{m}
\end{array}\right),
$$

where pi represents a subjective probability assigned to $x^{i}, i=1, \ldots, m$, by a given consumer according to his degree of belief in the occurrence of $x^{i}$ (De Finetti, 1982). We note that it turns out to be $\sum_{i=1}^{m} P_{i}=1$. We have then a coherent evaluation of the probabilities associated with every single random case. It is finitely additive. Different consumers whose state of knowledge is hypothetically identical may choose different pi whose sum is equal to 1 (Coletti, Petturiti, \& Vantaggi, 2016). Indeed, each of them may subjectively give greater attention to certain circumstances than to others (Coletti, Petturiti, \& Vantaggi, 2014). In any case, if we write

$$
(x, p) \subset E^{m}
$$

then we identify a distribution of probability embedded in a linear space (Pompilj, 1957). Such a distribution can always vary from consumer to consumer (De Finetti, 1975). Moreover, it can also vary with respect to the state of information of a given consumer (De Finetti, 1989). We also write

$$
u(\mathbf{x})=\left(\begin{array}{c}
u\left(x^{1}\right) \\
u\left(x^{2}\right) \\
\vdots \\
u\left(x^{m}\right)
\end{array}\right),
$$

where each $u\left(x^{i}\right)$ is the subjective utility of $x^{i}, i=1, \ldots, m$. It is a contravariant component of an $m$-dimensional vector of $E^{m}$. If we write

$$
(u(x), p) \subset E^{m}
$$

then we identify a distribution of probability embedded in a linear space, where every covariant component of $\mathrm{p}$ $2 \mathrm{Em}$ is a subjective probability assigned to $u\left(x^{i}\right), i=1, \ldots, m$. We will note that we deal with the same probabilities viewed as masses associated with $x^{i}$ and $u\left(x^{i}\right), i=1, \ldots, m$.

\section{A Contingent Consumption Plan Geometrically Decomposed Inside of a Linear Space}

We decompose a contingent consumption plan into $\mathrm{m}$ consumption bundles. We are also able to say that we decompose $X$ into $\mathrm{m}$ single events. We write

$$
X=x^{1}\left|E_{1}\right|+x^{2}\left|E_{2}\right|+\ldots+x^{m}\left|E_{m}\right|,
$$

where we have

$$
\left|E_{i}\right|= \begin{cases}1, & \text { if } E_{i} \text { is true } \\ 0, & \text { if } E_{i} \text { is false }\end{cases}
$$

for every $i=1, \ldots, m$ as well as $x^{i} \in \mathbb{R}, i=1, \ldots, m$. We consider m elementary events $E_{i}, i=1, \ldots, m$, of a finite partition of incompatible and exhaustive events. We observe that $\mathrm{X}$ is an identity function such that it is possible to write

$$
i d_{\mathbb{R}}: \mathbb{R} \rightarrow \mathbb{R}
$$

where $\mathbb{R}$ is a linear space over itself and it is of dimension 1 . We say that $X$ is a linear operator whose canonical expression coincides with (10). We say that $X$ is an isometry. It follows that each single event could uniquely be identified with infinite numbers, so we could also write $\left\{x^{1}+a, x^{2}+a, \ldots, x^{m}+a\right\}$, where $a \in \mathbb{R}$ is an arbitrary constant. This means that we consider infinite translations in this way. We consider different quantities from a geometric viewpoint. They are nevertheless the same quantity from a randomness viewpoint because events and probabilities associated with them do not change. On the other hand, if two or more than two propositions can express the same event contained in $X$ then two or more than two real numbers can identify it. We have to note a very important point: each contravariant component of $\mathrm{x}$ can be viewed as an $\mathrm{m}$-dimensional vector. It is denoted by ${ }_{(\mathrm{i})} \mathrm{x}, i=1, \ldots, m$. This vector and the corresponding vector of the orthonormal basis of $E^{m}$ denoted by $e_{i}, i=$ $1, \ldots, m$, are collinear. This is because there exists a real number denoted by $\alpha$ such that it turns out to be

$$
{ }_{(i)} \mathbf{x}=\alpha \mathbf{e}_{i},
$$

where we have $i=1, \ldots, m$. With regard to (13) we observe that $\alpha$ takes any value in $\mathbb{R}$. If we have $i=1$ in (13) then the vector $e_{l}$ identifies a straight line having a given direction in $E^{m}$. When $\alpha$ takes a value in $\mathbb{R}$ we note that $\alpha$ identifies an m-dimensional vector lying on the same straight line established by $e_{1}$ in $E^{m}$. We therefore say that this $m$-dimensional vector and $e_{1}$ are collinear. The same thing goes when $\alpha$ takes all values in $\mathbb{R}$. In 
particular, we observe that $\alpha$ always takes a value coinciding with the first contravariant component of $x$. We note that only the first component of $x$ in (3) is not equal to 0 . All other components of it are equal to 0 . We therefore write

$$
{ }_{(1)} \mathbf{x}=x^{1} \mathbf{e}_{1}
$$

If we have $i=2$ in (13) then the vector $e_{2}$ identifies a straight line having a given direction in $E^{m}$. This direction is orthogonal to the one of $e_{l}$. When $\alpha$ takes a value in $\mathrm{R}$ we note that $\alpha$ identifies an m-dimensional vector lying on the same straight line established by $e_{2}$ in $E^{m}$. We therefore say that this m-dimensional vector and $e_{2}$ are collinear. The same thing goes when $\alpha$ takes all values in $\mathbb{R}$. In particular, we observe that $\alpha$ always takes a value coinciding with the second contravariant component of $\mathrm{x}$. We note that only the second component of $\mathrm{x}$ in (3) is not equal to 0 . All other components of it are equal to 0 . We therefore write

$$
\text { (2) } \mathbf{x}=x^{2} \mathbf{e}_{2} \text {. }
$$

If we have $i=m$ in (13) then the vector em identifies a straight line having a given direction in $E^{m}$. This direction is orthogonal to the one of $e_{1}$. It is also orthogonal to the one of $e_{2}$ and so on until you get to $e_{m-l}$. When $\alpha$ takes a value in $\mathbb{R}$ we note that $\alpha$ identifies an m-dimensional vector lying on the same straight line established by em in $E^{m}$. We therefore say that this m-dimensional vector and em are collinear. The same thing goes when $\alpha$ takes all values in $\mathbb{R}$. In particular, we observe that $\alpha$ always takes a value coinciding with the $\mathrm{m}$-th contravariant component of $\mathrm{x}$. We note that only the m-th component of $x$ in (3) is not equal to 0 . All other components of it are equal to 0 . We write

$$
{ }_{(m)} x=x^{m} e_{m}
$$

Each state of nature is geometrically established by a straight line belonging to $E^{m}$. Each straight line of $E^{m}$ identifies an one-dimensional subspace of $E^{m}$ ((Pistone, Riccomagno, \& Wynn, 2001)). The direct sum of $m$ one-dimensional subspaces of $E^{m}$ coincides with Em itself so we write

$$
E_{(1)}^{m} \oplus \ldots \oplus E_{(m)}^{m}=E^{m},
$$

where each $E_{(i)}^{m}, i=1, \ldots, m$, denotes the $\mathrm{i}$-th one-dimensional subspace of $E^{m}$. We note that this direct sum is also orthogonal. Also, it turns out to be

$$
\operatorname{dim} E_{(1)}^{m}+\ldots+\operatorname{dim} E_{(m)}^{m}=\operatorname{dim} E^{m},
$$

where we have $\operatorname{dim} E^{m}=m$. After taking (17) into account we write

$$
{ }_{(1)} \mathbf{x}+\ldots+{ }_{(m)} \mathbf{x}=\mathbf{x},
$$

where each ${ }_{(i)} x$ is an element of $E_{(i)}^{m}, i=1, \ldots, m$, while $\mathrm{x}$ is an element of $E^{m}$. The contravariant components of (i) $x$ are given by

$$
{ }_{(i)} \mathbf{x}={ }_{(i)} x^{l} \delta_{i}^{J},
$$

where we have $i=1, \ldots, m$. We note that $\delta_{i}^{j}$ denotes the Kronecker delta. If it turns out to be $\mathrm{i}=\mathrm{j}$ then we have $\delta_{i}^{j}=1$. If it turns out to be $i, j$ then we have $\delta_{i}^{j}=0$. We note that (20) is characterized by the Einstein summation convention. Thus, we are able to write

$$
{ }_{(i)} \mathbf{x}=\left(\begin{array}{c}
{ }_{(i)} x^{1} \delta_{1}^{1}+{ }_{(i)} x^{2} \delta_{2}^{1}+\ldots+{ }_{(i)} x^{m} \delta_{m}^{1} \\
{ }_{(i)} x^{1} \delta_{1}^{2}+{ }_{(i)} x^{2} \delta_{2}^{2}+\ldots+{ }_{(i)} x^{m} \delta_{m}^{2} \\
\vdots \\
{ }_{(i)} x^{1} \delta_{1}^{m}+{ }_{(i)} x^{2} \delta_{2}^{m}+\ldots+{ }_{(i)} x^{m} \delta_{m}^{m}
\end{array}\right)
$$

where we have $i=1, \ldots, m$. We have to note another very important point: we say that we have $x^{1}<x^{2}<\ldots<x^{m}$ without loss of generality because we could indifferently choose any ordered m-tuple of straight lines of $E^{m}$. All these straight lines of $E^{m}$ are the axes of the coordinate system under consideration. A single state of nature is a particular random quantity. It follows that the probability of an event is conceptually contained in the prevision or expected value or mathematical expectation of a random quantity. The notion of prevision of a random quantity is a unique notion (Berti, Regazzini, \& Rigo,2001). It is called probability in the case of events. Hence, the same symbol $P$ is used in order to denote both the prevision of a random quantity and the probability of an event.

\section{The Expected Utility Function Viewed as a Vector Whose M Components Are All Equal}

It is possible to assign a further real number to every element Ei expressed by $x^{i}, i=1, \ldots, m$, belonging to one of $\mathrm{m}$ straight lines of $E^{m}$. It is denoted by $u\left(x^{i}\right), i=1, \ldots, m$. We observe that it turns out to be $u\left(x^{l}\right)<u\left(x^{2}\right)<\ldots<$ 
$u\left(x^{m}\right)$. All these numbers identify a utility function (Markowitz, 1987). They represent a way of describing subjective preferences. We note that a more preferred alternative gets assigned a larger number than a less preferred alternative. Each $u\left(x^{i}\right)$ is a numerical representative of $E_{i}, i=1, \ldots, m$. We say that $u\left(x^{i}\right), i=1, \ldots, m$, is a point of the corresponding straight line of $E^{m}$. We consequently observe that the expected utility function is given by

$$
\mathbf{P}(U)=U\left(x^{1}, \ldots, x^{m}\right)=\overline{\mathbf{x}}=\sum_{i=1}^{m} u\left({ }_{(i)} \mathbf{x}\right) p_{i},
$$

where we have $0 \leq P\left(E_{i}\right) \leq 1, i=1, \ldots, m$, and $\sum_{i=1}^{m} P_{i}=1$ because we consider a coherent evaluation of the probabilities related to the set of events expressed by $\left\{E_{l}, \ldots, E_{m}\right\}$. We consider the same events having the same probabilities viewed as masses. Only their numerical representatives are different. They coincide with $u\left(x^{l}\right), \ldots$, $u\left(x^{m}\right)$, where we have

$$
u\left({ }_{(i)} \mathbf{x}\right)=\left[u\left(x^{i}\right)\right] \mathbf{e}_{i}
$$

for every $i=1, \ldots, m$. We note that the Einstein summation convention does not hold with regard to (23). We say that (22) is an m-dimensional vector belonging to $E^{m}$ whose contravariant components are all equal. We note that the $\mathrm{i}$-th contravariant component of $\bar{x}$ is given by

$$
\bar{x}^{i}=u\left({ }_{(i)} x^{i} \delta_{i}^{j}\right) p_{i},
$$

where we have $i=1, \ldots, m$. We observe that it turns out to be $\mathrm{u}(0)=0$. Each contravariant component of $\bar{x}$ is then obtained by means of a linear combination. This linear combination is characterized by (24). We observe that the Einstein summation convention holds with regard to (24). Each contravariant component of $\bar{x}$ is then established by $m$ groups of numbers where every group of numbers consists of m numbers that are added. When we consider the first contravariant component of $\bar{x}$ we note that only the first element of the first group having $\mathrm{m}$ elements as summands is not equal to 0 . All other elements of the first group having $\mathrm{m}$ elements as summands are equal to 0 . When we consider the first contravariant component of $\bar{x}$ we note that only the second element of the second group having m elements as summands is not equal to 0 . All other elements of the second group having m elements as summands are equal to 0 . When we consider the first contravariant component of $\bar{x}$ we note that only the $\mathrm{m}$-th element of the $\mathrm{m}$-th group having $\mathrm{m}$ elements as summands is not equal to 0 . All other elements of the $m$-th group having $\mathrm{m}$ elements as summands are equal to 0 . The same thing goes when we consider all other contravariant components of $\bar{x}$. We observe that it turns out to be

$$
\mathbf{P}(X)=\overline{\overline{\mathbf{x}}}=\sum_{i=1}^{m}{ }_{i} \mathbf{x} p_{i},
$$

where all components of $\overline{\bar{X}} \in E^{m}$ are equal. We write

$$
\overline{\bar{x}}^{i}={ }_{(i)} x^{i} \delta_{i}^{j} p_{i}
$$

for every $i=1, \ldots, m$.

\section{The Expected Utility Function Geometrically Decomposed Inside of a Linear Space}

If we decompose a contingent consumption plan characterized by m possible consumption bundles then we are also able to decompose the expected utility function given by $U\left(x^{I}, \ldots, x^{m}\right)$ (Schoemaker, 1982). Given any one-dimensional subspace of $E^{m}$, the collinear vectors related to $U\left(x^{l}, \ldots, x^{m}\right)$ are two. We have

$$
e_{1}=1 \cdot e_{1}
$$

as well as

$$
u\left(_{(1)} \mathbf{x}\right)=\left[u\left(x^{1}\right)\right] \mathbf{e}_{1}
$$

with regard to the first one-dimensional subspace of $E^{m}$. A same probability denoted by $\mathrm{p} 1$ is associated with (28) even when $u\left(x^{l}\right)$ varies. In general, a same probability denoted by $p_{I}$ is associated with (28) when we consider $u\left(x^{l}\right)+\mathrm{a}$, where a is an arbitrary constant. We identify different m-dimensional vectors on a same straight line in $E^{m}$ in this way. The direction of this straight line is established by $e_{1}$. All these collinear vectors lying on the straight line established by $e_{1}$ represent the same event from a randomness viewpoint on condition that the starting inequalities given by $u\left(x^{I}\right)<u\left(x^{2}\right)<\ldots<u\left(x^{m}\right)$, where we have $\mathrm{a}=0$, continue to be valid in the form expressed by $u\left(x^{l}\right)+\mathrm{a}<u\left(x^{2}\right)+\mathrm{a}<\ldots<u\left(x^{m}\right)+\mathrm{a}$, where we have a $\neq 0$. We evidently consider a positive monotonic transformation in this way. This same event is then realized when the true value of $\left\{x^{1}, x^{2}, \ldots, x^{m}\right\}$ to be verified a posteriori coincides with the lowest possible value of $\left\{x^{l}, x^{2}, \ldots, x^{m}\right\}$. Conversely, we write 


$$
e_{m}=1 \cdot e_{m}
$$

as well as

$$
u\left(_{(m)} \mathbf{x}\right)=\left[u\left(x^{m}\right)\right] \mathbf{e}_{m}
$$

with regard to the $m$-th one-dimensional subspace of $E^{m}$. A same probability denoted by pm is associated with (30) even when $u\left(x^{m}\right)$ varies. In general, a same probability denoted by pm is associated with (30) when we consider $u\left(x^{m}\right)+\mathrm{a}$, where a is an arbitrary constant. We identify different $\mathrm{m}$-dimensional vectors on a same straight line in $E^{m}$ in this way. The direction of this straight line is established by em. All these collinear vectors lying on the straight line established by em represent the same event from a randomness viewpoint on condition that the starting inequalities given by $u\left(x^{l}\right)<u\left(x^{2}\right)<\ldots<u\left(x^{m}\right)$, where we have a $=0$, continue to be valid in the form expressed by $u\left(x^{l}\right)+\mathrm{a}<u\left(x^{2}\right)+\mathrm{a}<\ldots<u\left(x^{m}\right)+\mathrm{a}$, where we have a $\neq 0$. We evidently consider a positive monotonic transformation in this way. This same event is then realized when the true value of $\left\{x^{1}, x^{2}, \ldots, x^{m}\right\}$ to be verified a posteriori coincides with the highest possible value of $\left\{x^{1}, x^{2}, \ldots, x^{m}\right\}$. The same thing evidently goes when we consider all other one-dimensional subspaces of $E^{m}$ (von Neumann \& Morgenstern, 1947). Given any one-dimensional subspace of $E^{m}$ established by a straight line in Em, we are able to consider infinite scalars related to this straight line of $E^{m}$. These scalars coincide with the nonzero contravariant components of all the $\mathrm{m}$-dimensional collinear vectors with respect to one of the basis vectors. If a varies in $\mathbb{R}$ then there are infinite possible positive monotonic transformations that can theoretically be considered. We say that there are infinite possible utility functions that can theoretically be considered. It is then possible to move along every straight line of $E^{m}$ in order to consider them. The expected utility function always coincides with the direct sum of $m$ vectors related to $\mathrm{m}$ incompatible and exhaustive events. Such a direct sum is also orthogonal. These $\mathrm{m}$ vectors belong to m one-dimensional subspaces of $E^{m}$. An mdimensional vector belonging to $E^{m}$ is uniquely obtained by means of a linear combination of $m$ basis vectors. We denote it by $y$. The contravariant components of this m-dimensional vector are $\mathrm{m}$ scalars whose sum coincides with the expected utility function given by

$$
\mathbf{P}(U)=U\left(x^{1}+a, \ldots, x^{m}+a\right)=u\left(x^{i}+a\right) p_{i}=u\left(x^{1}+a\right) p_{1}+\ldots+u\left(x^{m}+a\right) p_{m} .
$$

We therefore write

$$
\left\{\left[u\left(x^{1}\right)+a\right] p_{1}\right\} \mathbf{e}_{1}+\ldots+\left\{\left[u\left(x^{m}\right)+a\right] p_{m}\right\} \mathbf{e}_{m}=\mathbf{y},
$$

where we have $y \in E^{m}$. Each of these $\mathrm{m}$ scalars is obtained by multiplying one of the $\mathrm{m}$ probabilities related to $\mathrm{m}$ incompatible and exhaustive states of nature by the contravariant component of the corresponding m-dimensional collinear vector belonging to one of the m one-dimensional subspaces of $E^{m}$.

\section{A Condition of Coherence Compatible with All Possible Attitudes in the Face of Risk of a Consumer}

The expected utility function has an additive structure (Markowitz, 1959). This means that the choices that a given consumer makes when a state of nature occurs are independent from the choices that he makes when another state of nature occurs, where one and only one state of nature occurs. This independence assumption is entirely caught by the linear independence of the basis vectors. A univariate random quantity representing all possible values of an investment denoted by $\mathrm{A}$ is expressed by $\mathrm{X}_{\mathrm{A}}$. A univariate random quantity representing all possible values of an investment denoted by $\mathrm{B}$ is expressed by $\mathrm{X}_{\mathrm{B}}$. We decompose $X_{A}=\left\{X_{A}^{1}, X_{A}^{2}, \ldots, X_{A}^{m}\right\}$, with $X_{A}^{1}<X_{A}^{2}<\cdots<X_{A}^{m}$, and $X_{B}=\left\{X_{B}^{1}, X_{B}^{2}, \cdots, X_{B}^{m}\right\}$, with $X_{B}^{1}<X_{B}^{2}<\cdots<X_{B}^{m}$, inside of $E^{m}$. We assign a number to every consumption bundle given by $X_{A}^{i}, \mathrm{i}=1, \ldots, \mathrm{m}$, and $X_{B}^{i}, \mathrm{i}=1, \ldots, \mathrm{m}$. It is denoted by $\mathrm{u}\left(X_{A}^{i}\right), \mathrm{i}$ $=1, \ldots, \mathrm{m}$, and $\mathrm{u}\left(X_{B}^{i}\right), \mathrm{i}=1, \ldots, \mathrm{m}$. We associate a probability denoted by $P_{i}^{A}$ with every $\mathrm{u}\left(X_{A}^{i}\right), \mathrm{i}=1, \ldots, \mathrm{m}$, where we have $P_{1}^{A}+\cdots+P_{m}^{A}=1$. These probabilities are the same of the ones associated with $X_{A}^{1}, X_{A}^{2}, \ldots, X_{A}^{m}$, because we deal with the same events. We associate a probability denoted by $P_{i}^{B}$ with every $\mathrm{u}\left(X_{B}^{i}\right), \mathrm{i}=1, \ldots, \mathrm{m}$, where we have $P_{1}^{B}+\cdots+P_{m}^{B}=1$. These probabilities are the same of the ones associated with $X_{B}^{1}, X_{B}^{2}, \ldots, X_{B}^{m}$, because we deal with the same events. It is therefore possible to calculate $\mathrm{P} U_{A}=\mathrm{u}\left(X_{A}^{1}, \ldots, X_{A}^{m}\right)=\bar{X}_{A}$, as well as $\mathrm{P} U_{B}=\mathrm{u}\left(X_{B}^{1}, \ldots, X_{B}^{m}\right)=\bar{X}_{B}$. It is also possible to calculate $\mathrm{P}\left(\mathrm{XA}_{\mathrm{A}}\right)=\overline{\bar{X}}_{A}$ as well as $\mathrm{P}\left(\mathrm{X}_{\mathrm{B}}\right)=\overline{\bar{X}}_{B}$. We consider 
their distances from the zero vector of $E^{m}$. For instance, we observe that if the components of $\bar{X}_{A}$ and $\bar{X}_{B}$ are positive then we strictly prefer $\bar{X}_{A}$ when it is more distant from the origin than $\bar{X}_{B}$. We mathematically write

$$
\left\|\bar{X}_{A}\right\|>\left\|\bar{X}_{B}\right\| \text {, }
$$

where $\left\|\bar{X}_{A}\right\|$ is the norm of $\bar{X}_{A}$ while $\left\|\bar{X}_{B}\right\|$ is the norm of $\bar{X}_{B}$. The same thing goes when we consider $\overline{\bar{X}}_{A}$ and $\bar{X}_{B}$. In general, one establishes if it turns out to be

$$
U\left(x_{A}^{1}, \ldots, x_{A}^{m}\right)>U\left(x_{B}^{1}, \ldots, x_{B}^{m}\right) \Longleftrightarrow\left\{x_{A}^{1}, \ldots, x_{A}^{m}\right\}>\left\{x_{B}^{1}, \ldots, x_{B}^{m}\right\}
$$

or

$$
U\left(x_{B}^{1}, \ldots, x_{B}^{m}\right)>U\left(x_{A}^{1}, \ldots, x_{A}^{m}\right) \Longleftrightarrow\left\{x_{B}^{1}, \ldots, x_{B}^{m}\right\}>\left\{x_{A}^{1}, \ldots, x_{A}^{m}\right\}
$$

or

$$
U\left(x_{A}^{1}, \ldots, x_{A}^{m}\right)=U\left(x_{B}^{1}, \ldots, x_{B}^{m}\right) \Longleftrightarrow\left\{x_{A}^{1}, \ldots, x_{A}^{m}\right\} \sim\left\{x_{B}^{1}, \ldots, x_{B}^{m}\right\} .
$$

The criteria of coherent decisions under uncertainty are all those consisting of the consideration of infinite utility functions and any coherent evaluation of the probabilities associated with every single random case. One of these utility functions must coherently be chosen (Johnson \& Payne, 1985). Moreover, the criteria of coherent decisions under uncertainty are all those by means of which one fixes as one's goal the maximization of the prevision of the utility associated with a contingent consumption plan (MacCrimmon, 1968). We say that a consumer coherently behaves in the face of risk when there exists an m-dimensional vector of Em uniquely obtained by means of a linear combination of $m$ basis vectors. We have

$$
\mathbf{z}=\left[u\left(x^{1}\right) p_{1}\right] \mathbf{e}_{1}+\ldots+\left[u\left(x^{m}\right) p_{m}\right] \mathbf{e}_{m},
$$

where it turns out to be $Z \in E^{m}$. The sum of the real coeffcients of this linear combination coincides with $u\left(x^{i}\right) p_{i}, i$ $=1, \ldots, m$. It is a mean value of $u\left(x^{i}\right), i=1, \ldots, m$. We propose a geometric condition of coherence compatible with all possible attitudes in the face of risk of a consumer. It is expressed by (37). It is appropriate to propose it because the attitude in the face of risk of a consumer could unexpectedly change. It depends on his temperament and his current mood. Moreover, it is also influenced by the value of his estate denoted by $F$. It is a random quantity because. This means that the true value of $F$ can be great or low. If the true value of $\mathrm{F}$ is unexpectedly great then his attitude in the face of risk may alter. The same thing goes if the true value of $\mathrm{F}$ is unexpectedly low (Slovic, Fischhoff, \& Lichtenstein, 1977). We do not write

$$
u(x)=u\left(x^{i}\right) p_{i}
$$

with $i=1, \ldots, m$, where (38) means that the right of getting $x^{1}$ associated with $E_{1}, \mathrm{x}^{2}$ associated with $E_{2}, \ldots, x^{m}$ associated with $E_{m}$, whose probabilities are expressed by $p_{1} ; p_{2}, \ldots, p_{m}$, is equal to the right of getting the certainty equivalent expressed by $x$. This is because we decompose the expected utility function inside of $\mathrm{E}_{\mathrm{m}}$, so we refer to infinite utility functions for which it turns out to be

$$
u\left(x^{l}\right)<u\left(x^{2}\right)<\ldots<u\left(x^{m}\right)
$$

Each of them could indifferently be a concave or convex or linear utility function.

\section{Monetary Value and Utility Coincide in the Case of a Risk-Neutral Consumer}

We get out of $E^{m}$. Let $u(x)$ be a continuous and strictly increasing utility function. It is a real-valued function having a real variable. By considering the inverse $u^{-1}(y)$ of $u(x)$ we obtain

$$
x=u^{-1}\left\{u\left(x^{i}\right) p_{i}\right\}
$$

When we deal with a risk-neutral consumer whose utility function denoted by $\mathrm{u}(\mathrm{x})$ is a linear function we note that $\mathrm{x}$ is equal to $P(X)=x^{l} p_{1}+\ldots+x^{m} p_{m}$. Such a utility function is a strictly increasing function whose increments (onto the $y$-axis) between $A$ and $x$ as well as between $x$ and $B$ are equal for a given consumer when and only when he is indifferent (onto the $x$-axis) between the choice of $x$, which is the certainty equivalent, and the choice of purchasing a lottery ticket connected with two random events. They are $A=$ "the ticket is not drawn" and $B=$ "the ticket is drawn", where $A$ and $B$ have equal probabilities. We have $\mathrm{P}(A+B)=\mathrm{P}(A)+\mathrm{P}(B)=1$ because $A$ and $B$ are incompatible and exhaustive events. This means that it turns out to be $\mathrm{P}(A)=\mathrm{P}(B)=\frac{1}{2}$. If $\mathrm{P}(X)=S$, where $S$ is an arbitrary monetary value which is different from 0 , then we are able to say that a risk-neutral consumer is indifferent between receiving with certainty $\mathrm{S}$ or $2 \mathrm{~S}=\mathrm{S}+\mathrm{S}$ in the case that $\mathrm{A}$ or $\mathrm{B}$ 
occurs. This means that $\mathrm{P}(X)=S$ coincides with $P(2 S A)=2 S P(A)=2 S_{2}^{1}=S$. If $\mathrm{P}(X)=-S$, where $-\mathrm{S}$ is an arbitrary loss which is different from 0 , then we are able to say that a risk-neutral consumer is indifferent between losing with certainty $-S$ or $-2 S=-(S+S)$ in the case that $A$ or $B$ occurs. This means that $P(X)=-S$ coincides with $\mathrm{P}(-2 \mathrm{SA})=-2 \mathrm{SP}(\mathrm{A})=-2 \mathrm{~S}_{2}^{1}=-\mathrm{S}$. We are also able to say that a risk-neutral consumer is indifferent between accepting or not accepting a bet for which it is possible for him to gain S if A occurs or to lose -S if B occurs. This means that 0 coincides with $\mathrm{P}(\mathrm{SA})+\mathrm{P}(-\mathrm{SB})=\frac{s}{2}-\frac{s}{2}=0$. It is evidently possible to extend what we have said. For instance, if $\mathrm{P}(\mathrm{X})=2 \mathrm{~S}$ then we are able to say that a risk-neutral consumer is indifferent between receiving with certainty $2 \mathrm{~S}$ or $4 \mathrm{~S}=\mathrm{S}+\mathrm{S}+\mathrm{S}+\mathrm{S}$ in the case that $\mathrm{A}$ or $\mathrm{B}$ occurs. This means that $\mathrm{P}(\mathrm{X})=2 \mathrm{~S}$ coincides with $\mathrm{P}(4 S A)=4 S P(A)=4 S_{\frac{1}{2}}^{1}=2 S$. It follows that $\mathrm{P}$ is additive, where $\mathrm{P}$ is nothing but a subjective price. Thus, $\mathrm{P}(X)$ is the prevision or mathematical expectation or price of $\mathrm{X}$ for a risk-neutral consumer. We therefore assume that if a risk-neutral consumer is willing to consume $\mathrm{n}$ different random quantities denoted by $X_{1}, \ldots, X_{n}$ at $\mathrm{n}$ prices denoted by $\mathrm{P}\left(X_{1}\right), \ldots, \mathrm{P}\left(X_{n}\right)$ then he is willing to consume them all together, where their price coincides with the sum of these $\mathrm{n}$ prices. We write $\mathrm{P}\left(X_{1}+\ldots+X_{n}\right)=\mathrm{P}\left(X_{I}\right)+\ldots+\mathrm{P}\left(X_{n}\right)$. If the criterion of the mathematical expectation is applied to the scale of utility coinciding with the monetary one then we say that among decisions under uncertainty leading to different random quantities a risk-neutral consumer chooses that random quantity having the highest prevision or mathematical expectation or price. If the scale of utility coincides with the monetary one then we write $u(S)=S$. We say that a risk-neutral consumer is rigid with respect to risk because we assume that $\boldsymbol{P}$ is additive.

\section{Conclusions}

A univariate random quantity representing a contingent consumption plan has been studied inside of $E^{m}$ because utility and probability are both subjective. We have considered distributions of probability embedded in a linear space provided with a Euclidean metric on it. We have replaced a closed structure with another one: we have replaced a $\sigma$-algebra witha linear space over $\mathbb{R}$. We have studied coherent decisions under uncertainty having as their goal the maximization of the prevision of the utility associated with a contingent consumption plan. We have studied the criterion of the mathematical expectation when it is applied to utility and monetary values. When it is applied to monetary values we have observed that among decisions under uncertainty leading to different random quantities a consumer chooses that random quantityhaving the highest prevision or mathematical expectation. When it is applied to the notion of utility we have considered the independence assumption as an implicit condition of coherence. This assumption is entirely caught by the linear independence of the vectors of an orthonormal basis of $E^{m}$. We have observed that a risk-neutral consumer coherently chooses a linear utility function as well as he coherently chooses his probabilities associated with every single random case. He does not choose what it is necessary in order to be coherent. This thing cannot arbitrarily be chosen. We have proposed a geometric condition of coherence compatible with all possible attitudes in the face of risk of an individual because we have measured utility inside of Em by taking $u\left(x^{l}\right)<\ldots<u\left(x^{m}\right)$ into account. We have consequently decomposed $u(x)$ inside of $E^{m}$ in this way.

\section{References}

Barlow, R. E., Claroti, C. A., \& Spizzichino, F. (1993). Reliability and decision making. Boca Raton: CRC Press. https://doi.org/10.1007/978-1-4899-4459-7

Battaglia, F. (2007). Metodi di previsione statistica. Springer Verlag, Milano. https://doi.org/10.1007/978-88-470-0603-4

Berti, P., Pratelli, L., \& Rigo, P. (2015). Two versions of the fundamental theorem of asset pricing. Electronic Journal of Probability, 20, 1-21. https://doi.org/10.1214/EJP.v20-3321

Berti, P., Regazzini, E., \& Rigo, P. (2001). Strong previsions of random elements. Statistical Methods and Applications (Journal of the Italian Statistical Society) 10, 11-28. https://doi.org/10.1007/BF02511636 
Coletti, G., \& Scozzafava, R. (2002). Probabilistic logic in a coherent setting. Dor-drecht/Boston/London: Kluwer Academic Publishers. https://doi.org/10.1007/978-94-010-0474-9

Coletti, G., Petturiti, D., \& Vantaggi, B. (2014). Bayesian inference: The role of coherence to deal with a prior belief function. Statistical Methods \& Applications, 23(4), 519-545. https://doi.org/10.1007/s10260-014-0279-2

Coletti, G., Petturiti, D., \& Vantaggi, B. (2016). Conditional belief functions as lower envelopes of conditional probabilities in a finite setting. Information Sciences, 339, 64-84. https://doi.org/10.1016/j.ins.2015.12.020

Coletti, G., Petturiti, D., \& Vantaggi, B. (2016). When upper conditional probabilities are conditional possibility measures. Fuzzy Sets and Systems, 304, 45-64. https://doi.org/10.1016/j.fss.2016.02.015

Coletti, G., Scozzafava, R., \& Vantaggi, B. (2015). Possibilistic and probabilistic logic under coherence: default reasoning and System P. Mathematica Slovaca, 65(4), 863-890. https://doi.org/10.1515/ms-2015-0060

De Finetti, B. (1972). How to choose the initial probabilities. In B. de Finetti (Ed.), Probability, Induction and Statistics (The art of guessing) (pp. 143-146). J. Wiley, London-New York-Sydney-Toronto.

De Finetti, B. (1972). Probability, Statistics and Induction: their relationship according to the various points of view. In B. de Finetti (Ed.), Probability, Induction and Statistics (The art of guessing) (pp. 147-227). J. Wiley, London-New York-Sydney-Toronto.

De Finetti, B. (1975). Theory of probability. J. Wiley \& Sons, 2 voll., London-New York-Sydney-Toronto.

De Finetti, B. (1980). Probability: beware of falsifications!. In H. E. Kyburg jr., \& H. E. Smokler (Eds.), Studies in subjective probability (pp. 195-224). Huntington, New York: R. E. Krieger Publishing Company.

De Finetti, B. (1981). The role of "Dutch Books" and of "proper scoring rules". The British Journal of Psychology of Sciences, 32, 55-56. https://doi.org/10.1093/bjps/32.1.55

De Finetti, B. (1982). Probability: the different views and terminologies in a critical analysis. In L. J. Cohen, J. Ło's, H. Pfeiffer, \& K. P. Podewski (Eds.), Logic, Methodology and Philosophy of Science VI, (pp. 391-394). Amsterdam: North-Holland Publishing Company. https://doi.org/10.1016/S0049-237X(09)70208-3

De Finetti, B. (1982). The proper approach to probability. In G. Koch, \& F. Spizzichino (Eds.), Exchangeability in Probability and Statistics (pp. 1-6). North-Holland Publishing Company, Amsterdam.

De Finetti, B. (1989). Probabilism: A Critical Essay on the Theory of Probability and on the Value of Science. Erkenntnis, 31(2/3), 169-223. https://doi.org/10.1007/BF01236563

DeGroot, M. H. (1962). Uncertainty, information and sequential experiments. The Annals of Mathematical Statistics, 33, 404-419. https://doi.org/10.1214/aoms/1177704567

Elton, E. J., \& Gruber, M. J. (1995). Modern portfolio theory and investment analysis. New York: Wiley \& Sons.

Feller, W. (1966). An introduction to probability theory and its applications. J. Wiley \& Sons, 2 voll., New York, I (1957); II.

Gilio, A., \& Sanfilippo, G. (2014). Conditional random quantities and compounds of conditionals. Studia logica, 102(4), 709-729. https://doi.org/10.1007/s11225-013-9511-6

Good, I. J. (1962). Subjective probability as the measure of a non-measureable set. In E. Nagel, P. Suppes, \& A. Tarski (Eds.), Logic, Methodology and Philosophy of Science (pp. 319-329). Stanford: Stanford University Press.

Jeffreys, H. (1961). Theory of probability (3rd ed.). Oxford: Clarendon Press.

Johnson, E. J., \& Payne, J. W. (1985). Effort and accuracy in choice. Management Science, 31(4), 395-414. https://doi.org/10.1287/mnsc.31.4.395

Kendall, M. G., \& Stuart, A. (1961). The advanced theory of statistics (vol. 2.). C. Griffin \& Co., London

Koopman, B. O. (1940). The axioms and algebra of intuitive probability. Annals of Mathematics 41, 269-292. https://doi.org/10.2307/1969003

Kreps, D. M. (1990). Game theory and economic modelling. Oxford: Clarendon Press. https://doi.org/10.1093/0198283814.001.0001

Kyburg jr., H. E., \& Smokler, H. E. (1964). Studies in subjective probability. New York, London, Sydney: J. 
Wiley \& Sons.

Levy, H., \& Sarnat, M. (1986). Capital investment and financial decisions. Prentice Hall, New York.

Lindley, D. V. (1965). Introduction to probability and statistics from a Bayesian viewpoint. I, Probability; II, Inference. London: Cambridge Univ. Press. https://doi.org/10.1017/CBO9780511662973

MacCrimmon, K. R. (1968). Descriptive and normative implications of the decision-theory postulates. In K. Borch, \& J. Mossin (Eds.), Risk and Uncertainty (pp. 3-32). London: Palgrave Macmillan. https://doi.org/10.1007/978-1-349-15248-3_1

Markowitz, H. M. (1959). Portfolio selection: Efficient diversification of investments. New York: Wiley \& Sons.

Markowitz, H. M. (1987). Mean-variance analysis in portfolio choice and capital markets. Basil Blackwell Inc., New York.

Mas-Colell, A., Whinston, M. D., \& Green, J. R. (1995). Microeconomic theory. New York: Oxford University Press.

McCullagh, P. (1987). Tensor methods in statistics. Chapman and Hall, London-New York.

Pfanzagl, J. (1967). Subjective probability derived from the Morgenstern-von Neumann utility theory. In M. Shubik (Ed.), Essays in mathematical economics in honor of Oskar Morgenstern (pp. 237-251). Princeton: Princeton University Press. https://doi.org/10.1515/9781400877386-020

Piccinato, L. (1986). De Finetti's logic of uncertainty and its impact on statistical thinking and practice. In P. K. Goel, \& A. Zellner (Eds.), Bayesian Inference and Decision Techniques (pp. 13-30). North-Holland, Amsterdam.

Pistone, G., Riccomagno, E., \& Wynn, H. P. (2001). Algebraic statistics. Chapman \& Hall, Boca Raton-London-New York-Washington, D.C. https://doi.org/10.1201/9781420035766

Pompilj, G. (1957). On intrinsic independence. Bulletin of the International Statistical Institute, 35(2), 91-97.

Ramsey, F. P. (1960). The foundations of mathematics and other logical essays. In R. B. Braithwaite with a preface by G. E. Moore. Littlefield, Adams \& Co, Paterson, N. J.

Savage, L. J. (1954). The foundations of statistics. New York: J. Wiley \& Sons.

Schoemaker, P. J. H. (1982). The expected utility model: its variants, purposes, evidence and limitations. Journal of Economic Literature, 20(2), 529-563.

Slovic, P., Fischhoff, B., \& Lichtenstein, S. (1977). Behavioral decision theory. Annual Review of Psychology, 28, 1-39. https://doi.org/10.1146/annurev.ps.28.020177.000245

Taylor, L. D. (1974). Probability and mathematical statistics. Harper \& Row, New York.

Von Neumann, J., \& Morgenstern, O. (1947). Theory of games and economic behavior (2nd ed.). Princeton: Princeton University Press.

\section{Copyrights}

Copyright for this article is retained by the author(s), with first publication rights granted to the journal.

This is an open-access article distributed under the terms and conditions of the Creative Commons Attribution license (http://creativecommons.org/licenses/by/4.0/). 